\title{
Un caso di destrocardia con Situs Viscerum Inversus Totalis concordante in coppia di gemelle DZ
}

\author{
Bondavalli W., Bondavalli G., La Bella G., Nizzoli A. \\ Assistenti
}

L'osservazione monocasistica nello studio dei gemelli ha un valore minore dell'indagine pluricasistica, anche se, sommando più osservazioni, si può giungere a risultati e ad interpretazioni sovente interessanti. Scrive infatti Gedda a pag. 996 del suo «Studio dei gemelli» (I) che "non bisogna sottovalutare, nè trascurare l'osservazione monocasistica, la quale merita di essere attentamente studiata, protocollata, pubblicata o altrimenti segnalata».

Durante le indagini schermografiche condotte dal Dispensario Antitubercolare di Reggio Emilia nella popolazione e nelle comunità organizzate della Provincia sono stati relativamente frequenti i casi di destrocardia repertati, specialmente associata a Situs Inversus Totalis. La trasposizione dei visceri non è infatti fenomeno eccezionale e sembra abbastanza frequente nelle sue varie espressioni: teoricamente è possibile ammettere che dal situs solitus si possa passare per gradi al Situs Inversus Totatis. Infatti la clinica ha potuto segnalare molti di questi quadri intermedi di trasposizione viscerale.

Già Aristotele (2) sapeva di una inversione della posizione della milza e del fegato da osservazioni su animali; al dire di Plinio (3) gli aruspici nei visceri degli animali riscontravano spesso anomalie di questo genere. È nel xviII secolo che si cominciò ad indagare sulle destrocardie e sulle inversioni viscerali ed il Riolano (4) nel i649 pubblicò un lavoro sul Situs Viscerum Inversus Totalis: egli emise tutta une serie di teorie con le quali volle chiarire con barocche spiegazioni le inversioni, presentando così il fianco alla ironia di Molière (5) e di Leibniz (6). Egli affermò che ai visceri invertiti corrispondesse una inversione morale: questi pregiudizi durarono poi a lungo. A suffragio di queste ipotesi errate si portò, ad esempio, il caso dell'assassino Francœur che presentava Situs Inversus Totalis dei visceri. Si affermò anche che Maria de' Medici avesse gli intestini completamente invertiti. Meyer (7) nel 1864 trova le conseguenze morali di una inversione, senza però affermare un rapporto certo fra una posizione irregolare degli organi ed una cattiva condotta. Krieger, nel I88o fa giustizia di queste interpretazioni. Bisogna arrivare al ${ }_{1} 928$ per trovare la prima descrizione di una destrocardia non complicata, corredata da un quadro radiografico ed ectografico, nella magistrale monografia di Mandelstamm e Reinberg (8). 
Schmidt e Korth (9) trattono diffusamente questo argomento e, premesso che spesso le destrocardie fanno parte del quadro più vasto del situs viscerum inversus totalis, concludono classificando le destrocardie in tre tipi (inversione, destroversione e destroposizione) secondo la posizione dei sistemi circolatori arterioso e venoso del cuore, non secondo l'architettura anatomica del cuore, anche se ad ognuna di queste forme fondamentali appartiene una architettura tipica.

I. Nell'inversione i sistemi circolatori sono immagini speculari del cuore normale (destrocardia così detta "à miroir ») (vedi fig. I.).

2. Nella destroversione i sistemi circolatori sono posti in modo che il ventricolo arteriale è sempre in posizione anteriore.

3. Nella destroposizione la topografia del sistema circolatorio è uguale al situs solitus.

Una domanda molto interessante che gli studiosi si sono posti è la seguente: l'inversione concerne tutto l'uomo o soltanto i visceri?

Geoffroy - Saint Hilaire ( 10 ) distinse una inversion génerale ed una inversion splanchnique. Nelle lumache, spesso citate a questo proposito, l'inversione si manifesta in un decorso speculare delle volute del guscio. Ma è facile obbiettare che l'uomo è costruito in modo molto simmetrico cosicchè non è possibile provare una inversion génerale.

La frequenza del situs viscerum inversus è varia secondo i diversi Autori e ciò è comprensibile: molti--studiosi raccoglievano il loro materiale statistico in grandi cliniche dove logicamente i portatori di situs inversus potevano essere accolti per le frequenti malformazioni associate alla anomalia; gli Autori che si interessavano di questi studi erano poi considerati degli specializzati in questa materia e gli ammalati venivano a loro convogliati. E quindi giusto ritenere con Schmidt e Korth (9) che le statistiche in proposito non sono molto attendibili. Con l'estendersi delle indagini schermografiche avremo certamente statistiche più precise. Le Wald (I I) nelle reclute trovava lo $0,0028 \%$ (è questa la percentuale più bassa di tutta la letteratura); nella sua pratica privata riscontrò lo $0,07 \%$ (è questa invece la percentuale più alta di tutta la letteratura). Mandelstamm e Reinberg (8) a Pietroburgo repertarono lo $0,076 \%$. Schmdit e Korth (9) in un grande complesso industriale tra gli operai ed i loro congiunti trovarono lo o,o125\%. Per quanto riguarda i due sessi la maggioranza degli Autori ritiene essere l'anomalia più frequente negli uomini che nelle donne (Gutmann (I2) 2,5: I per gli uomini; Gruber (I3) 2,6: (I); Küchenmeister (I4) 2: I).

Circa la familiarità e la ereditarietà del situs inversus i pareri sono discordi: Leininger e Gibson (15) soltanto nel $10 \%$ dei casi della letteratura accertarono il situs inversus familiare. Per Cockaine (i6) l'eredità del situs inv. totalis è recessiva e l'inversione viene probabilmente decisa da un unico gene.

Per Schmidt e Korth (9) il situs inv. totalis è una malformazione ancorata al genotipo.

Il fenomeno presenta poi una particolare distribuzione geografica: Tardos (I 7) riferisce le seguenti percentuali: Vietnam, frequenza dello $0,3 \%$, Africa del Nord $0,2 \%$; Europa 0,08\%. 


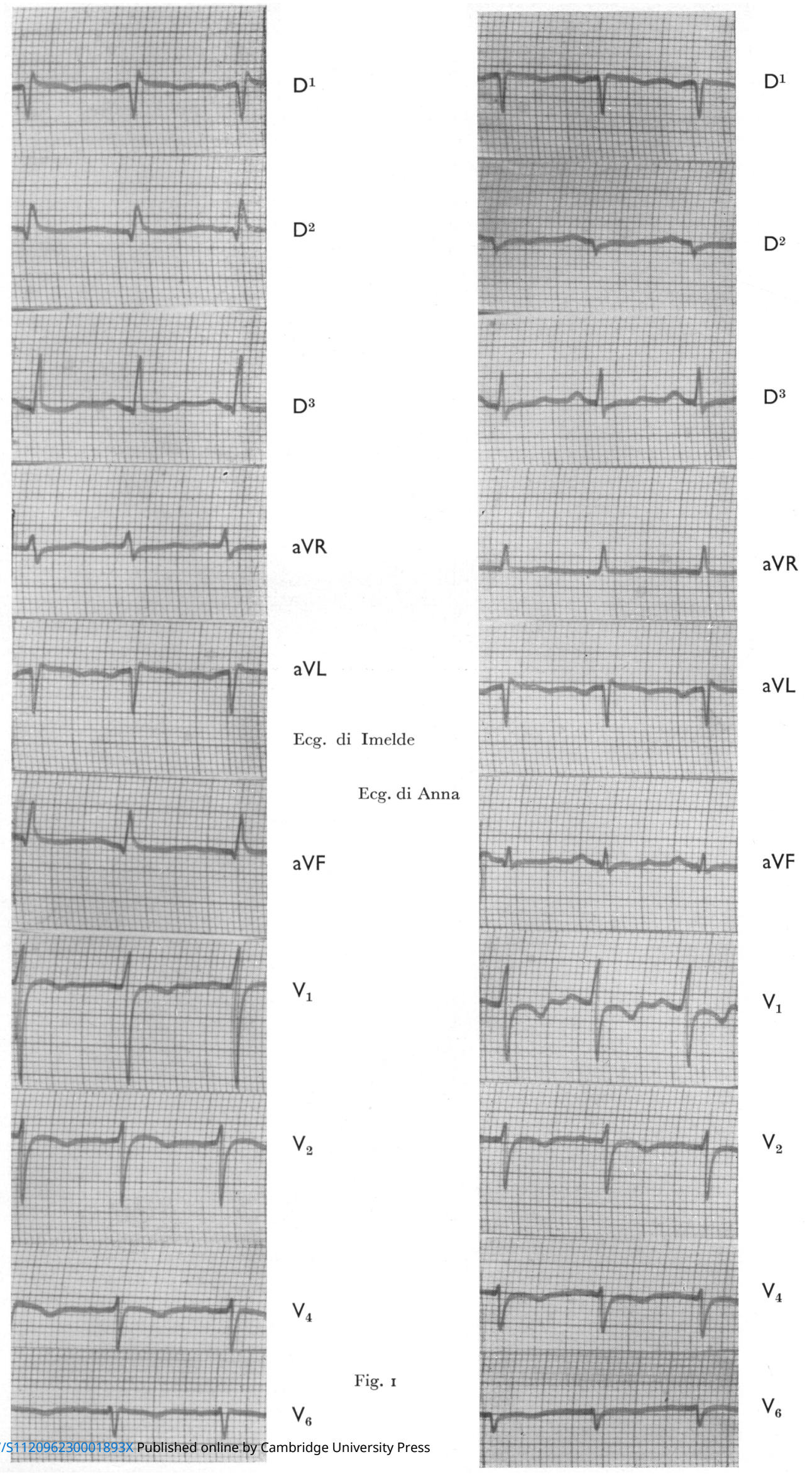


Se dunque l'inversione dei visceri sembra essere fenomeno relativamente frequente, rare e sempre molto interessanti sono le comunicazioni riguardanti tale malformazione nei gemelli.

Destrocardia o situs inversus sono stati segnalati in un solo gemello di una coppia, « così talora avviene che un gemello presenti fenomeni di lateralità invertita rispetto al suo cogemello» (Gedda, op. cit. pag. 489).

Questi contributi sono importantissimi nel campo della gemellologia, perchè riguardano il capitolo affascinante della specularità. Casi di inversione sperimentale sono segnalati dalla embriologia sperimentale come nella esperienza di Dareste (18) (1877) che otteneva il situs inversus mediante riscaldamento di uova fecondate: ricorderemo le più recenti esperienze di Morgan (19) sulle uova di ascidia le quali, quando vengono isolati i due primi blastomeri, presentano gastrulazioni in ciascun blastomero con fenomeni di specularità a carico delle cellule muscolari; Spemann (20) nel Triton taeniatus otteneva artificialmente «duplicitas anterior» con situs viscerum inversus in un gemello coalescente. Nella gemellarità umana si incontrano diversi fenomeni di specularità che Zazzo (21), seguendo Bouterwerk (22), chiama col nome di «miroir physique».

Araki (23) nel i 935 descrisse una coppia di gemelli monozigoti giapponesi nella quale un membro (A) della coppia presentava il situs inv. viscerum totalis, accertato clinicamente, radiologicamente e con l'elettrocardiogramma; il padre, che aveva avuto questi gemelli dalla prima moglie ne ebbe un'altra coppia dalla seconda moglie, ma normali; il gemello A era anche mancino e presentava situs inversus scrotalis; il cogemello era normale e destrimane.

Specularità intrageminali da situs v. inv. totalis di un solo membro di coppia gemellare MZ furono segnalate anche da Miller (24) (I893) e da Cockaine (16) (I950). Altri casi furono individuati nella letteratura da Newman (25) (1940), il quale cosi si esprime: "Per quanto abbiamo potuto scoprire vennero sinora pubblicati solamente 5 casi di gemelli umani $\mathrm{MZ}$ separati che presentavano situs viscerum inversus in uno dei membri gemellari. Questo fatto veniva scoperto durante l'autopsia di gemelli nati morti o morti durante l'infanzia ».

La gemellarità teratologica, cioè le formazioni doppie, fornisce cospicui fenomeni di specularità. Nei fratelli siamesi xifopaghi Eng e Ciang (Allen e Pancoast, I875) (26) si notava destrocardia in un membro e situs solitus del cuore nell'altro; così pure nelle toracopaghe Maria e Rosalina secondo Chapot e Prevost (I9or) (27). Küchenmeister (14) (I888) nell'autopsia di due coppie toracopaghe, trovò una cavità toracica comune, unico pericardio e dentro a questo i due cuori con l'asse diretto l'uno verso sinistra e l'altro verso destra.

Il situs inversus può riguardare soltanto il cuore: allora prende il nome di destrocardia isolata congenita e quando si verifica in un solo gemello offre un altro esempio di specularità intrageminale. Casi del genere furono descritti da Schott (28) (189I) nei riguardi di una coppia MZ di 45 anni e da Paltauf (rgo I) che segnalò destrocardia 
Bondavalli W., Bondavalli G., La Bella G., Nizzoli A.: Un caso di destrocardia, ecc.

in un solo feto di una coppia MZ di sette mesi. Più recentemente Ostertag e Spaich (30) (1935) descrissero la coppia Berta ed Elisabetta di 28 anni nella quale solo Elisabetta presentava destrocardia: ella era un'ottima sportiva nonostante la sua destrocardia ed il suo rendimento sportivo era maggiore di quello della cogemella con situs cordis solitus.

Destrocardie e situs inversus concordanti in gemelli sono ancora più rari: per quanto ci è stato possibile rintracciare nella bibliografia, soltanto quattro casi sono stati finora pubblicati. Reinhardt (3I) (I9I2-I3) descrive una coppia costituita da due gemelli MZ di 20 anni i quali, in occasione della visita medica per il servizio militare, dimostrano una trasposizione dei visceri totale e concordante; un caso analogo fu descritto in Italia da Pezzi e Carugati nel ig24 (32).

Boccia e Maglione (1927) (33) in Sudamerica osservarono e descrissero un altro caso di situs inversus concordante in gemelli. Kean nel $194^{2}$ (34) osservò concordanza di situs inversus nelle gemelle di una coppia MZ di 18 anni: una era affetta da endocardite lenta. Torgersen (35) nel 1948 descrisse una coppia di gemelli $\mathrm{DZ}$, maschi entrambi con situs inversus concordante; ma in un membro della coppia si trattava di situs inv. totalis, mentre nel cogemello c'era solo situs inv. abdominalis. Matisson (36) ( I933) studiando il problema dell'eredità del situs inversus totalis cercò di mettere in evidenza $\mathbf{i}$ rapporti di questo fenomeno con la gemello-parità; mentre non gli fu possibile riscontrare una frequenza di nascite gemellari più alta del normale nelle famiglie tarate dall'inversione, analizzando con il metodo differenziale di Weinberg le nascite gemellari di quattro famiglie tarate trovò una frequenza aumentata di coppie MZ e cioè quattro coppie che corrispondevano al $50 \%$ del totale delle nascite gemellari, anzichè al $25-30 \%$ come di norma. Quindi Matisson, pur riconoscendo la necessità di elaborare un materiale più vasto, è propenso ad ammettere "una correlazione positiva tra la frequenza della inversione viscerale e la frequenza della gemelliparità MZ» (citato da Gedda).

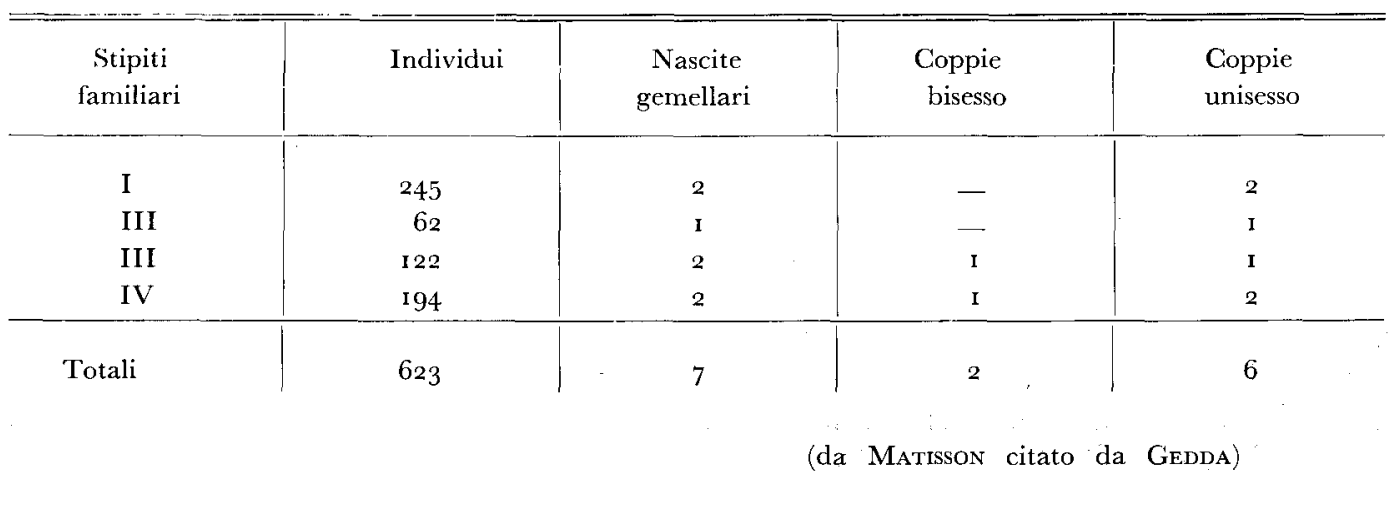


Il caso che è occorso alla nostra osservazione è costituito da una coppia di gemelle $\mathrm{DZ}$ (vedi fig. 3) con situs inversus totalis concordante. Ci è parso degno di segnalazione, perchè, per quanto ci è stato possibile accertare, è questo il quinto caso descritto dopo i casi di inversione concordante di Reinhardt, di Pezzi e Carugati, di Boccia e Maglione e di Kean ed il primo di tale anomalia concordante in gemelli DZ. Le gemelle B. Imelde e Anna risiedono a Vezzano sul Crostolo in provincia di Reggio Emilia: sono nate il 26 ottobre 1926 da genitori tuttora viventi e sani: il parto fu eutocico. La madre riferisce che il sacco coriale era unico. Dall'albero genealogico ririprodotto nella fig. 2 si desume che la loro è stata l'unica nascita gemellare nella famiglia. Hanno cinque fratelli viventi e sani e con situs normalis. Alla nascita Imelde pesava Kg. 2,300 e Anna Kg. 3,00o. Ebbero allattamento materno, i primi atti fisici e psichici furono normali, hanno frequentato la terza classe elementare.

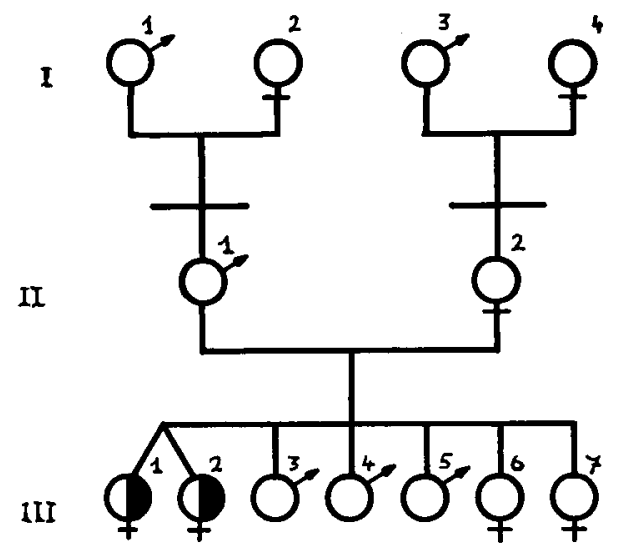
I. I. B. Pietro $\nmid 70$ a. (ictus)
2. P. Giuseppina $\nmid 92$ a. (senectus)
3. G. Guglielmo +86 a. (senectus)
4. B. Elvira +65 a. (polmonite)
II. I. B. Pompeo vivente situs solitus
2. G. Maria vivente situs solitus

III. I. Imelde s. visc. inv.

2, Anna s. visc. inv.

3. Piero situs solitus

4. Nello situs solitus

5. Severo situs solitus

6. Nella situs solitus

7. Alda situs solitus

Fig. 2 - Albero genealogico

Il rendimento scolastico delle due gemelle fu normale: erano ambedue deboli in matematica. La prima mestruazione si manifestò in ambedue a 12 anni.

Anna sposò a 22 anni ed ebbe due figli, Imelde sposò a 25 anni ed ebbe un figlio: i figli sono normali. Lavorano come contadine; il loro rendimento di lavoro è sempre stato ottimo: modiche mangiatrici, non fanno uso di vino non gradiscono cibi grassi. Imelde pesa $52 \mathrm{Kg}$. ed Anna 50: Imelde è più espansiva ed allegra, Anna più riservata e timida. Non hanno sofferto malattie degne di nota se si eccettuano $i$ comuni esantemi dell'infanzia. Imelde ha subito recentemente un intervento chirurgico per correzione del setto nasale: ha sofferto in passato di numerosi episodi tonsillitici.

Abbiamo anzitutto proceduto alla diagnosi di zigotismo secondo il metodo di 
Bondavalli W., Bondavalli G., La Bella G., Nizzoli A.: Un caso di destrocardia, ecc.

Siemens-von Verschuer (Polysymptomatische Aehnlichkeits-Diagnose): compilata la scheda di Gedda abbiamo notato notevoli discordanze per quanto concerne l'altezza, il perimetro toracico, i diametri cranici: discordanti sono i caratteri del polso, della pressione arteriosa, della temperatura. Concordanti viceversa risultano i caratteri della pelle per ciò che riguarda il colore, l'aspetto, la lanugo ed il dermografismo: per l'impronte digitali (vedi fig. 4) si rileva concordanza per 3 dita corrispondenti (mano destra I, 4; mano sinistra 4). Ricordiamo a questo proposito che Stocks (37) presuppone che in gemelli di egual sesso si tratti di MZ se in almeno sette dita corrispondenti delle mani si trova concordanza o specularità.

Per i capelli abbiamo trovato concordanza per l'impianto, per il vortice, discordanza per il colore (più chiaro in Anna). Von Verschuer (39) trovò concordanza del colore dei capelli nel $75,8 \%$ dei MZ e solo nel $7 \%$ dei DZ.

Per i caratteri degli occhi abbiamo rilevato semplice somiglianza delle sopracciglia e delle palpebre, concordanza del colore dell'iride (azzurro chiaro): Il visus è in entrambi di ı/ı. Notevoli discordanze si sono potute mettere in evidenza per i caratteri delle labbra, della cavità orale e dei denti. Esistono significative differenze nella forma dell'arcata dentaria e dei denti. Imelde presenta in modo evidente $\mathrm{i}$ canini superiori più sporgenti che in Anna. Imelde presenta inoltre ipertrofia delle tonsille palatine. Discordanti sono i caratteri dei padiglioni auricolari, del trago; del lobulo. Anna presenta maggiore lunghezza del naso, è stata sottoposta a correzione del setto. Discordanti sono anche i caratteri sessuali secondari. Sono ambedue destrimani.

I risultati dell'esame immunoematologico, eseguito dal Prof. Rosalino Sacchi del Policlinico di Bologna, offrono i seguenti risultati:

Imelde: $A_{1}, M N$, GcDee $(R h)$

Anna: $A_{1} B, M N$, CcDee $(R h)$

I risultati del del dosaggio del glutatione ematico furono i seguenti:

Imelde: Glutatione Ridotto mmgr. 31,6\%

Glutatione Ossidato " $\quad 4,4 \%$

Glutatione Totale $\quad " \quad 36 \%$

Anna: Glutatione Ridotto mmgr. 21,5\%

Glutatione Ossidato " $" 5,5 \%$

Glutatione Totale " $\quad 27 \%$

Aggiungeremo che le due gemelle in parola non mostrano in complesso caratteri somatici tali da farle classificare come gemelle identiche. Ci siamo dilungati nel sottolineare i caratteri discordanti che ci permettono di porre diagnosi di dizigotismo: abbiamo fatto questo di proposito perchè il caso da noi descritto non deve, a nostro parere, essere considerato alla stregua di una semplice curiosità scientifica. La dimostrazione di casi di situs inversus concordante in gemelli dizigoti può offrire un contributo alla soluzione della vexata quaestio se ai gemelli $\mathrm{DZ}$ debba essere negato o concesso il carattere di vera gemellanza. Siemens nel ig24 affermava che «ogni 

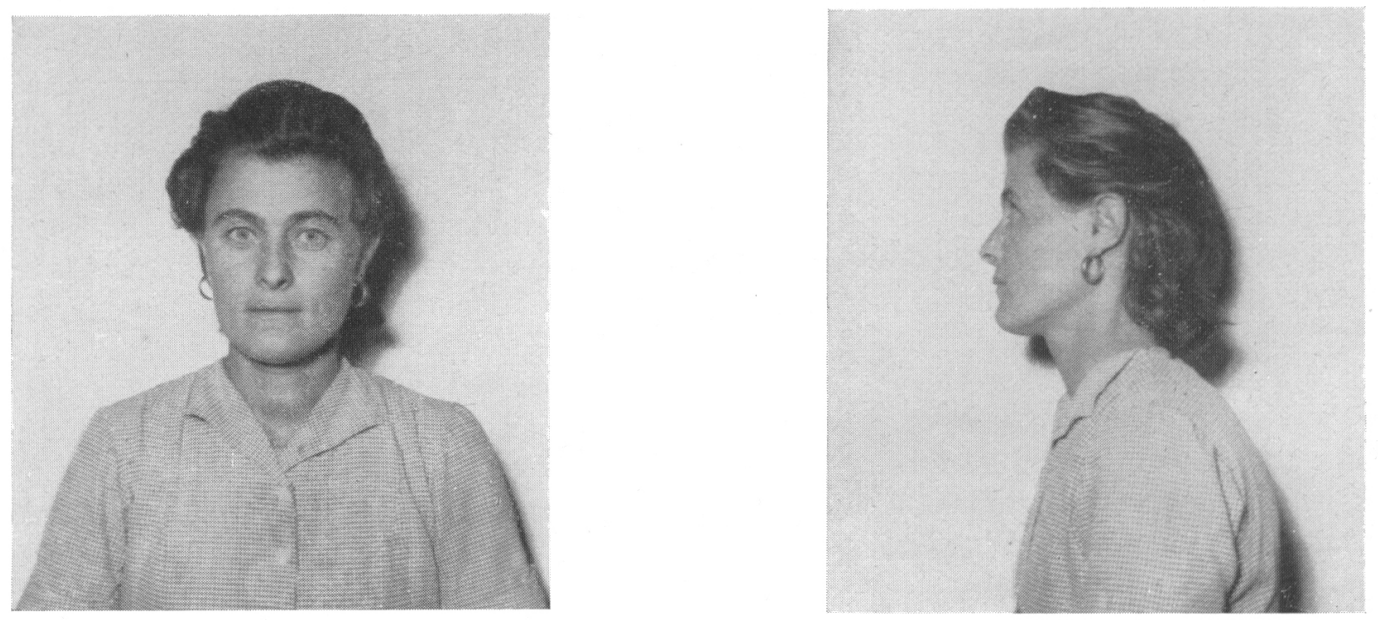

Fig. 3a - Fotografie e radiografia toracica di Imelde B.

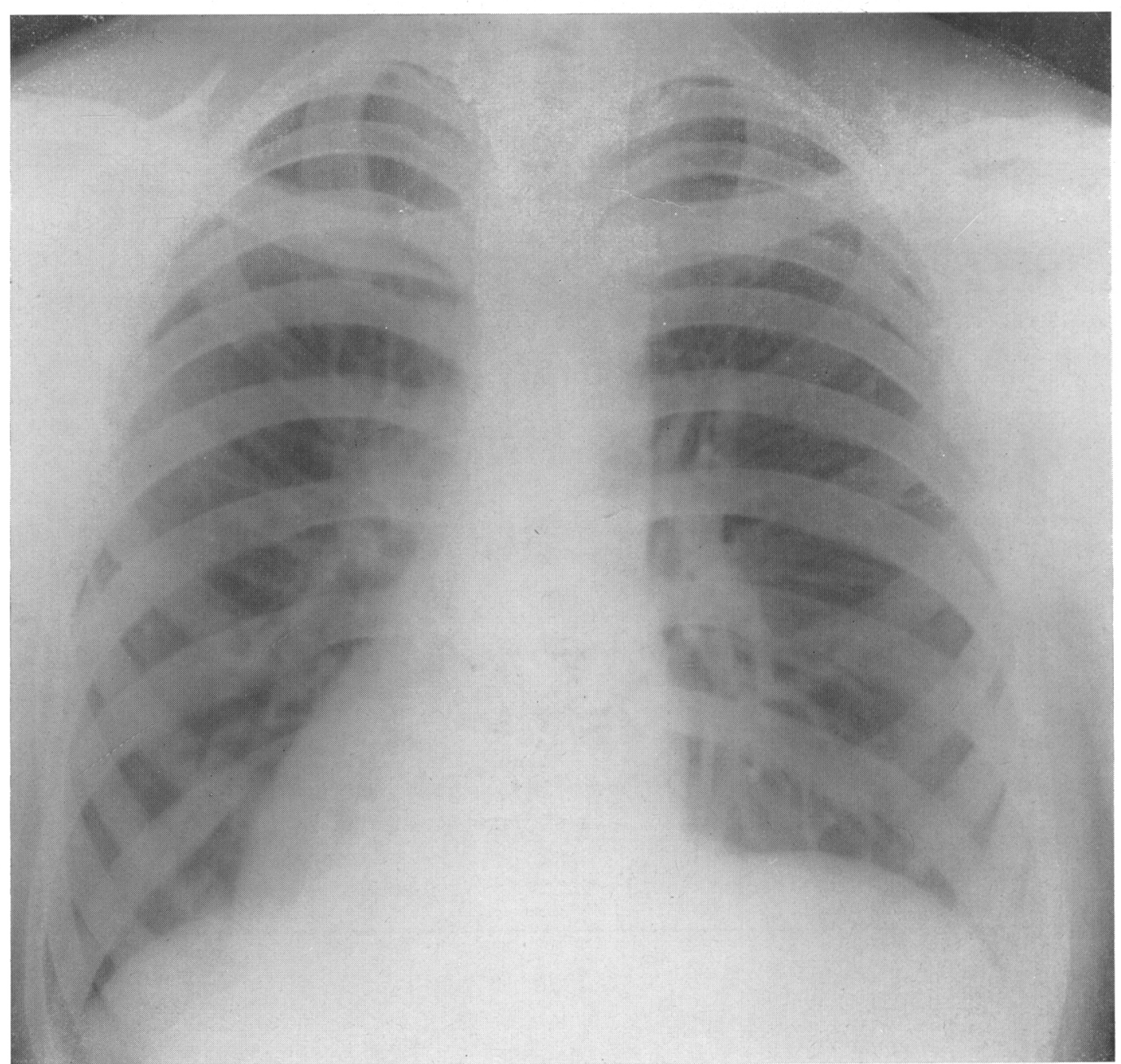



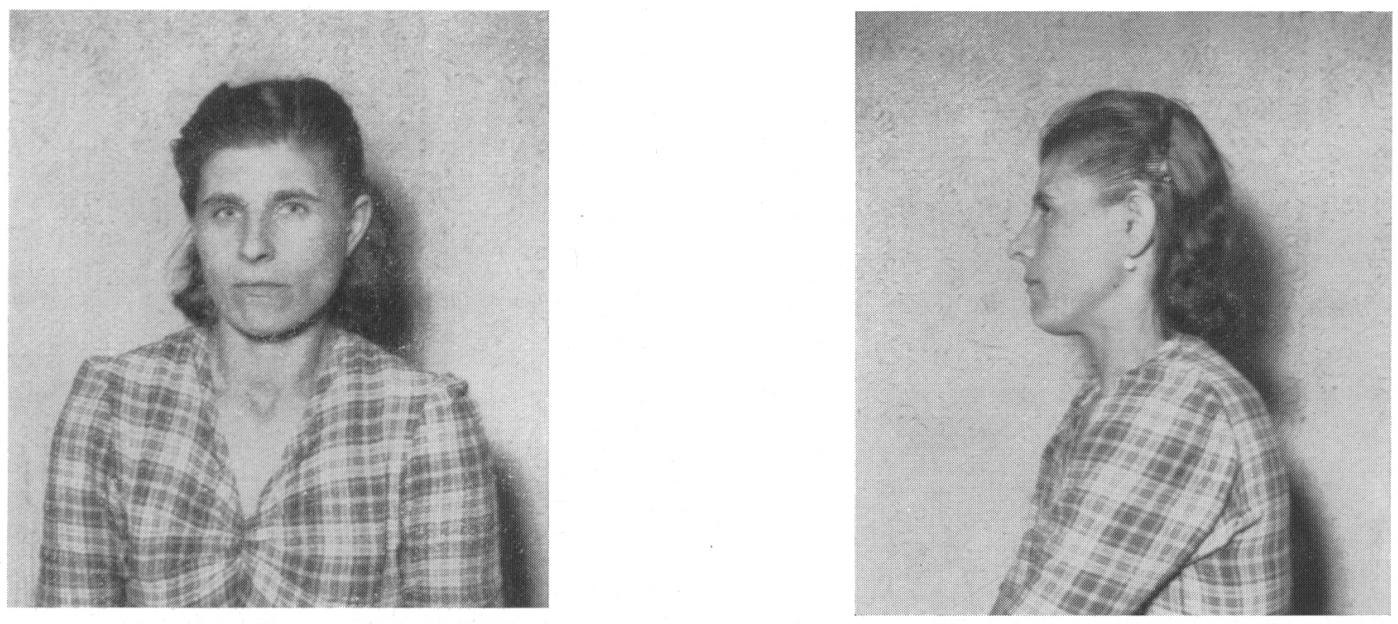

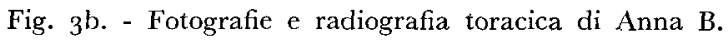

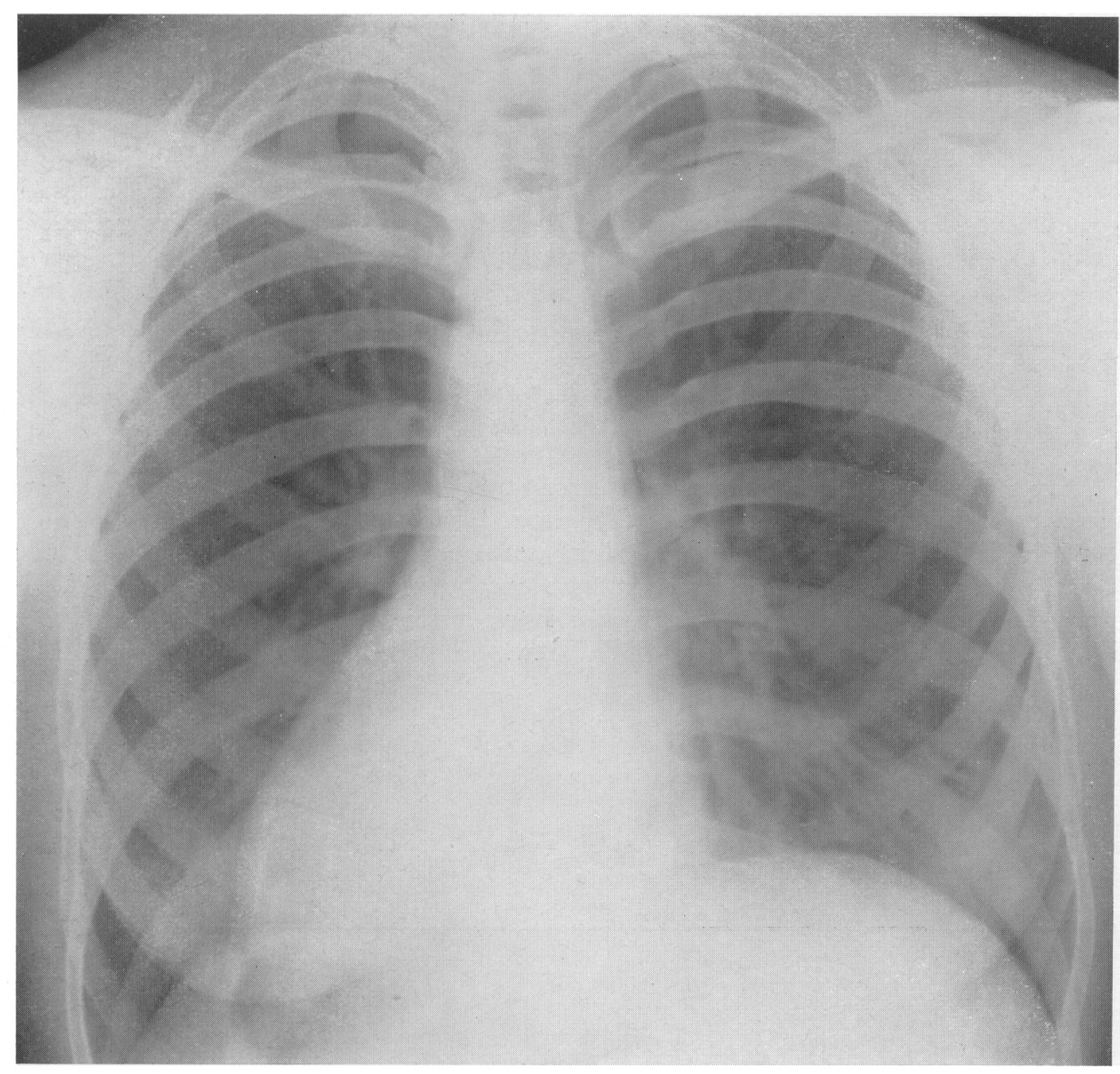


affezione ereditaria viene osservata contemporaneamente molto più spesso in gemelli identici, che non in gemelli non identici, ed in questi ultimi più spesso che non tra fratelli». Diversi autori infatti considerano i cosiddetti gemelli falsi o fraterni (dizigoti) alla stregua di semplici fratelli, colmando lo scarto fra gemelli DZ e fratelli non gemelli con la considerazione che i primi affrontano contemporaneamente le medesime condizioni ambientali. «In altri termini, lo scarto fenotipico fra le due categorie di gemelli viene attribuito ad uno scarto mesologico postnatale, e si tende ad escludere l'intervento di fattori endogeni, ereditari, costituzionali, come fattori determinanti della discriminazione fenotipica fra gemelli DZ e fratelli non gemelli, riconoscendo invece allo scarto che li distingue il valore di un fenomeno peristatico" (Gedda, op. cit. pag. 377).

Considerazioni di ordine dottrinale ed il comportamento della glutationemia gemellare portano giustamente Gedda a concludere che, non escludendo la componente mesologica, questa non sia l'unica causa a porre per cosi dire i gemelli DZ a mezza strada fra la concordanza dei gemelli $\mathrm{MZ}$ e la discordanza assoluta dei fratelli non gemelli.

Se fosse vero che tutta l'eredità è retta dal meccanismo mendeliano, cioè cromosomico, nucleare, si potrebbe con tranquillità equiparare i gemelli $\mathrm{DZ}$ ai fratelli non gemelli, perchè gli uni e gli altri costruiscono in modo analogo il loro fenotipo. Oggi si intravvede che il protoplasma dei gameti giuoca un ruolo importante nei fenomeni ereditari e si parla giustamente di una quota di eredità citoplasmatica. D'altro canto altri meccanismi non strettamente ereditari potrebbern conferire ai gemelli DZ una affinità biologica maggiore di quella posseduta dai fratelli non gemelli: alcuni Autori italiani (Oggioni, Tarozzi) parlano infatti da tempo di "congenicità ". Tarozzi nel I9I 8 scriveva: "l'aver avuto in comune la vita intrauterina è cosa di tale importanza da essere sufficiente a far sì che una grande differenza corra tra due gemelli e tra fratelli provenienti da diverse gravidanze ».

Il caso da noi descritto di inversione dei visceri nelle sole gemelle DZ Anna e Imelde, mentre gli altri fratelli non gemelli presentano situs solitus, non è spiegabile con la circostanza che le gemelle furono concepite pressapoco contemporaneamente e meno ancora per il fatto che si svilupparono simultaneamente nel corpo materno. Si deve riconoscere che in questo caso tra le due gemelle, nonostante la discordanza di molti caratteri, c'è una affinità biologica infinitamente più alta che non tra $i$ fratelli non gemelli. A meno che non si voglia ritenere che la spinta alla inversione dei visceri sia da ricercare in particolari condizioni intrauterine (e ciò non pare sostenibile) bisogna riconoscere alla gemellanza DZ il carattere di vera ed autentica gemellanza. 


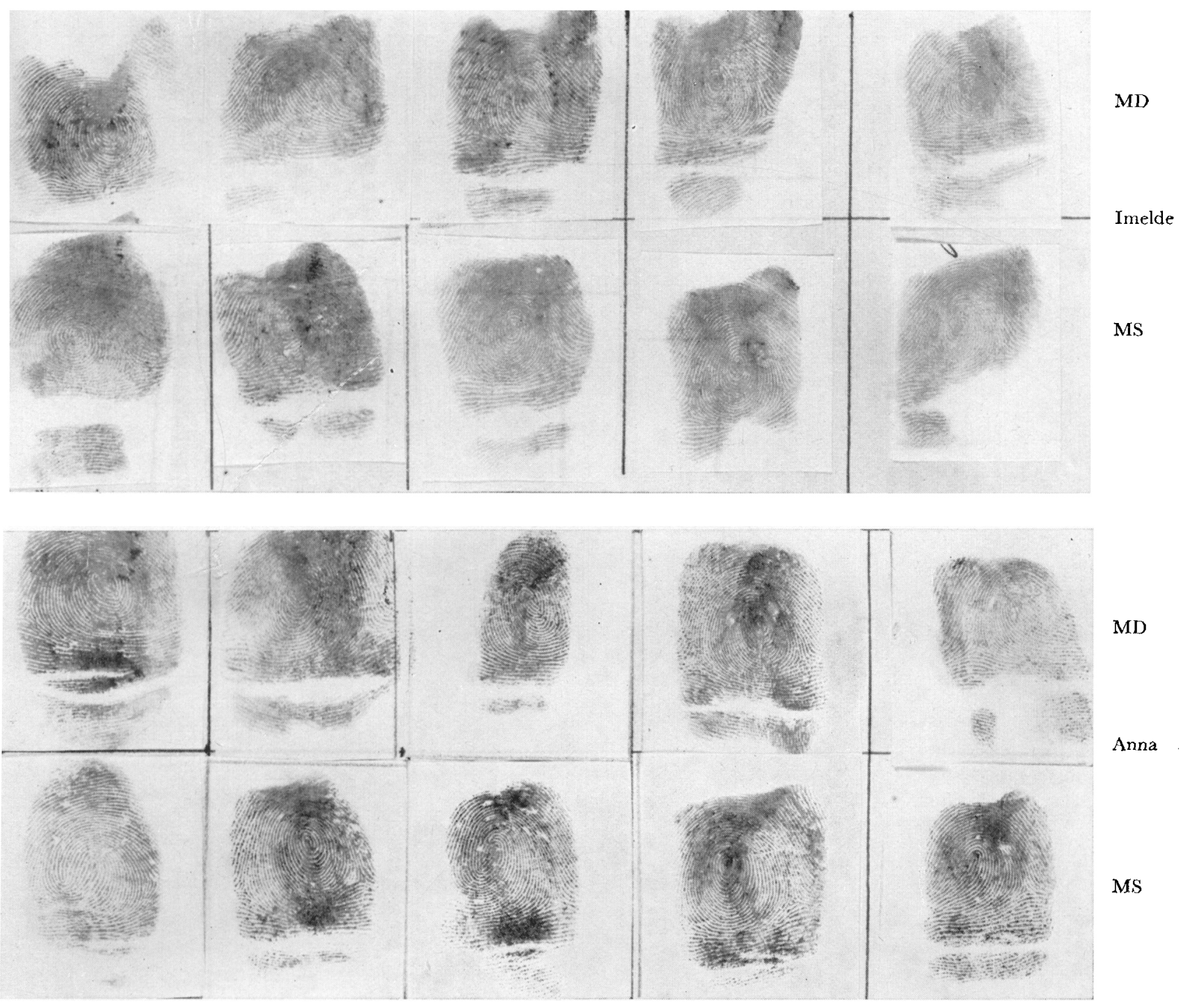

Lianw le sorelle

Liomo Le s-arelle Beneventi. Alice Benvents. Ymelole
Ymelde thang

Fig. 4. - Impronte digitali e calligrafia di Imelde e Anna B. 


\section{Riassunto}

Gli autori descrivono un caso di situs viscerum inversus totalis in una coppia di gemelle DZ. Discutono, in base agli elementi in loro possesso, se sono di fronte a ge. melle DZ o MZ e concludono per la prima ipotesi. Così questo è il quinto caso descritto di tale anomalia in gemelli dopo i casi di Reinhardt, Pezzi e Carugati, Boccia e Maglione, Kean, ed il primo in gemelli DZ. Ritengono che questo caso contribuisca a riconoscere alla gemellanza $\mathrm{DZ}$ dignità di vera gemellanza in accordo alle asserzioni di Gedda. 


\section{Bibliografia}

I. Gedda, L.: Studio dei gemelli. Ed. Orizzonte. Medico. Roma 195I.

2. Aristotele: De generatione animalium. Lib. IV, Cap. IV.

3. Plinio: Naturalis historia, XI, 204.

4. Riolano, J.: Opusc. Anat. varia et nova, S. 117 ff. Parigi 1652.

5. Molière: Citato da Schmidt e Korth.

6. Leibniz: Citato da Schmidt e Korth.

7. Meyer: Citato da Schmith e Korth.

8. Mandelstamm, M. e Reinberg, S.: Erg. inn. Med. Kinderheilk., 34, i54 (i928).

9. Schmidt, J. e Korti, C.: Arch. für Kreislaufforschung., 2 I, 188, I 954.

io. Geoffroy St. Hilaire, I.: Histoire générale et particuliére des anomalies de l'organisation chez l'homme et les animaux, Bd. I I, 5, 2 1, Parigi. I 836.

I1. LE WALD, L. T.: J.A.M.A., 84:26I (1925)

12. Guttmann: Berliner Klin. Wschr. i 5 o, i 876 .

13. Gruber, W.: Arch. Anat. Physiol. Wissenschaftl. Med., 558, r865.

14. Küchenmeister, F.: Die Angeborene, vollstindige seitliche Verlagerung der Eingeweide des Menschen. Lipsia $188_{3}$.

15. Leininger, C.R. e St. Gibbson, J.: Pediatr., 37: i95 (I950).

16. Cockayne, E.: Quart. J. Med., 7: 479, 1937.

17. Takdos, M.: A propos des inversions viscérales totales. Réunions Med-Chirurg. de Saigon-Cholon, I4 avril I950.

18. Dareste, G.: Compt. rend. Acad. d. Sc. Paris io4, 715-717, i887.

19. Morgan, Th. H.: Embriologia e genetica. Torino $193^{8 .}$

20. Spemann, H. e Falkenberg, H.: Arch. f. Entw. Med., 45:371-422; 19 I8.

21. Zazzo, M.R.: Echos de Med., I4: 7, 4, r943.

22. Bouterwek, H.: Ztschr. f. menschl. Vererb. u. Konstitutioslehre, 2 I : 5, 737-760, $193^{8}$.

23. Araki, Bungo: Nagasaki Ygakkwai Zassi., i 3: I6gi-i 7oo, 1935 -

24. Miller, N.F.: Homologishe Zwillinge. Jahrb. f. Kinderh., 36: 333, 1893 .

25. Newwman H, Freeman, Frank N., Holzingze, Karl J.: Human Biol., 12 : 2 1-34, 1940.

26. Allen e Pancoast, citati da Gedda.

27. Chapot Prevost, E.: Chirurgie des tèratopages; operation de Maria-Rosalina. Observations d'un nouveau xiphopage, les frères chinois. Parigi rgor.

28. Schott, citato da Gedda.

29. Paltauf, R.: Wien. Klin. Wsch., ro32, rgor.

3o. Ostertag, M. e Spaif, D.: Ztschr. f. menschl, Vererb. u. Konstitutionslehre., i9: 5, 577-584, i953.

31. Reinilardt, Z.: Dtsch. militärarztl. Zeit., 41: 932, 19 I2.

- Deutsche med. Wschnschr, 82: I9I3.

32. Pezzi, C. e Carugati, L.: Cuore e circolazione., 8: 36 r, ig24.

33. Boccia, e Maglione, R.: Rev. sudamericana endr.cr., io: 705, r927.

34. KeAn, B.H.: J. Hered., 33: 6. 21 7-221, I942.

35. Torgersen, J.: J. Hered., 39: io; 293-294, 1948.

36. Matisson, K.: Z. Konstitutionslehre, 17: 325, 1933.

37. Stocks, T.: Am. of Eugen. I, I I. 4, 49-108, 1930.

$3^{8}$. V. Verschuer, citato da Gedda.

39. Siemens, H.W.: Die Zwillingspathologie. Monaco 1924. 
The authors describe a case of " situs viscerum inversus totalis ") in two $\mathrm{DZ}$ twin-sisters.

They debate, on the basis of the elements in their possession, whether they have to do with $\mathrm{DZ}$ or MZ twin-sisters, and declare in favour of the former hypothesis. Accordingly, this is the fifth case described of such anomaly in twins, after the cases of Reinhardt, Pezzi and Carugati, Boccia and Maglione, Kean, and it is the first case in $\mathrm{DZ}$ twins.

The authors think the case described will contribute in winning for $\mathrm{DZ}$ twinship an acknowledgement of real twinship, according to Gedda's statements.
Les auteurs donnent la description d'un cas de " situs viscerum inversus totalis" constaté en deux sœurs jumelles DZ.

Se fondant sur les éléments en leur possession, les auteurs discutent s'ils ont affaire à des sœurs jumelles $\mathrm{DZ}$ ou bien $\mathrm{MZ}$, et ils se déclarent favorables à la premiére hypothèse. Il s'agit du cinquième cas de cette anomalie dans les frères jumeaux, dont on donne la description, après les cas de Reinhardt, Pezzi et $\mathrm{Ca}$ rugati, Boccia et Maglione, Kean, et le premier observé en des jumeaux DZ.

L'opinion des auteurs est que ce cas ne peut que contribuer à faire reconnaître aux jumeaux DZ, la qualité de véritables jumeaux, d'après les affirmations de Gedda.

\section{ZUSAMMENFASSUNG}

Die Verfasser beschreiben einen Fall von "Situs viscerum inversus totalis " in einem zweieiigen weiblichen Zwillingspaar. Auf Grund der in ihrem Besitz befindlichen Elemente überlegen die Verfasser, ob sie es mit ZZ oder EZ zu tun haben und neigen dabei mehr zu ersterer Hypothese. Nach den von Reinhardt, Pezzi u. Carugati, Boccia u. Maglione, Kean beschriebenen Fällen ist dies der fünfte Fall und der erste bei $\mathrm{ZZ}$.

Die Autoren denken, dass dieser Fall dazu beitragen wird, die zweieiigen Zwillingsbildungen in Übereinstimmung mit Gedda's Behauptungen als echte Zwillingsbildungen zu würdigen. 